\title{
Intelligent technologies help operating mobile cabin hospitals effectively cope with COVID-19
}

(C) Higher Education Press 2020

Coronavirus disease 2019 (COVID-19) has spread fast, and the number of confirmed cases worldwide reaches 167511, in which 81077 are recorded in China until March 16, 2020 (WHO, 2020a). In response to the situation, the Chinese government has marshalled the full resources and efforts of the country to reduce the COVID-19 morbidity and mortality. As the number of confirmed cases decreases considerably every day, China's control approach for COVID-19 epidemic has been proven to be agile and productive (National Health Commission, 2020; WHO, 2020b). Among all interventions, operating Chinese mobile cabin hospitals is considered one of the most effective measures because it holds many patients simultaneously at the outbreak stage; as such, this strategy quickly and effectively contains the spread of the virus (WHO, 2020b; Zhang, 2020b).

Chinese government has built 16 mobile cabin hospitals (Fig. 1) in 2 weeks (Wen et al., 2020) and rehabilitated more than 12000 patients with no medical staff infected (Tian et al., 2020). Valuable experiences are gained from the course, especially in using intelligent technologies for preventing cross infections and addressing the shortage of health care workers when operating mobile cabin hospitals.

First, Building Information Modeling (BIM) tools can be used to optimize the space structure, layout and construction equipment in mobile cabin hospitals. High

Received April 1, 2020

Ying ZHOU, Lingling WANG, Lieyun DING

Center for Smart and Healthy Buildings, Huazhong University of Science and Technology, Wuhan 430074, China

\section{Zhouping TANG ( $($ )}

Department of Neurology, Tongji Hospital, Tongji Medical College, Huazhong University of Science and Technology, Wuhan 430030, China E-mail: ddjtzp@163.com structure space is the basic premise of the mobile cabin hospital because it can decrease the risk of virus spreading through the air. In China, large-span buildings, such as industrial plants and stadiums, were selected to be renovated into mobile cabin hospitals. At most, 3000 patients can be accommodated in the hospital, for which, effective measures should be considered to prevent cross infections. BIM tools were used to simulate instantaneous airflow and pollutant dispersion and optimize the space arrangement of mobile cabin hospitals to keep from the spread of infections. In addition, a distributed indoor environmental monitoring system was recommended to improve the operation of ventilation systems and purification equipment.

Second, using Internet-of-Things (IoT) technologies for contactless management should be considered as much as possible to cope with limited healthcare services and prevent the spread of infections among patients and medical staff. In Chinese mobile cabin hospitals, each health care worker who manages an average of 50 patients bears a heavy psychological and physiologic burden (Zhang, 2020a). Smart devices can become important alternatives of some manual workload. For instance, intelligent robots were used in some mobile cabin hospitals to perform some transportation activities, such as drug delivery and garbage disposal. Wristbands can also be applied to regularly and automatically collect physical indicators, such as temperature and blood oxygen, of each patient. Wearable technologies can trace patients' behavioral trajectories in real time. If a patient enters a prohibited area, such as a medical waste area, then the patient and management personnel can be warned or reminded in accordance with the activity information.

Third, a cloud-based system has been established to improve the operation efficiency of hospital and the treatment effects of COVID-19 and support better treatment plans. This system can record all the collected basic, clinical physiologic, and medical imaging information of 


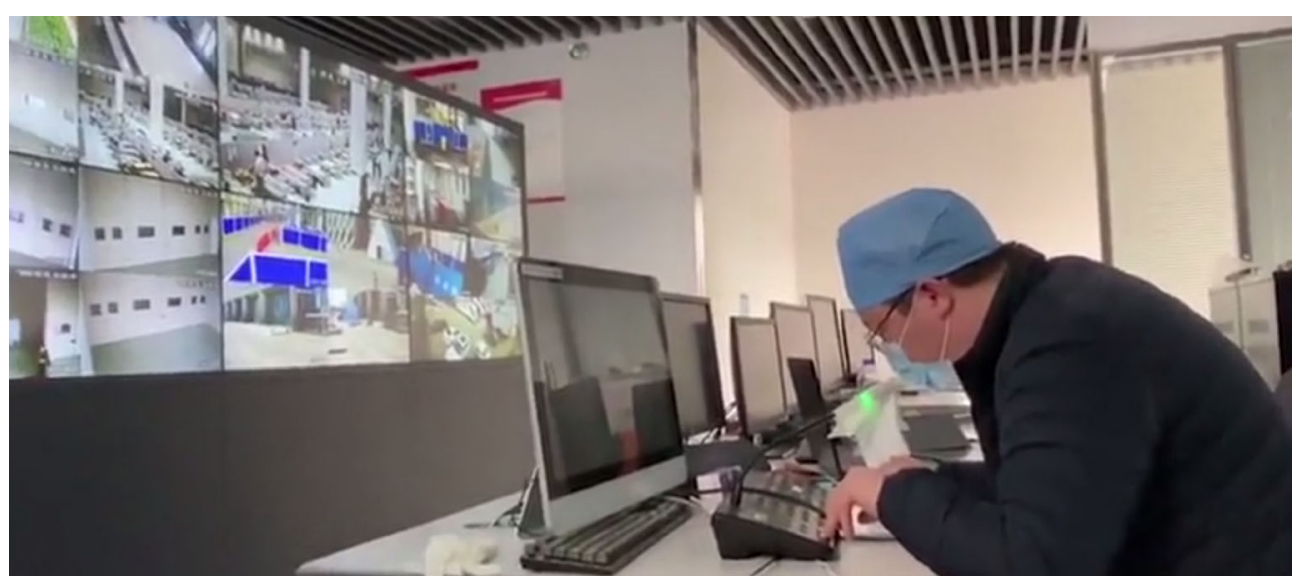

Fig. 1 Intelligent optical valley mobile cabin hospital.

each patient. These data can be analyzed for accurate diagnosis and scientific medication. The data can also be shared with experts for remote consultation. All the information can be saved for future use to form a big data system for better understanding the COVID-19 virus, including its pathogenesis and therapy.

Mobile cabin hospitals play an irreplaceable role in China's battle against COVID-19. Isolating and taking care of patients timely are still the most effective measures in the absence of a well-proven specific vaccine or drug for coronavirus. To overcome the shortage of medical resources and high risk of cross infection, intelligent technologies have taken irreplaceable role when operating the mobile cabin hospitals. In the past 10 days, COVID-19 has swept through more than 100 countries, causing dozens of increases in the number of confirmed cases outside China (WHO, 2020c). Experiences learned from the operation of mobile cabin hospitals can provide guidance for China in responding to other major public health emergencies in the future as well as valuable insights for coping with the COVID-19 epidemic in other affected countries.

\section{References}

National Health Commission (2020). March 17: Daily briefing on novel coronavirus cases in China. National Health Commission of the People's Republic of China. Available at: en.nhc.gov.cn/2020-03/17/ c 77822.htm

Tian D D, Wu S, Wu J (2020). Wuhan mobile cabin hospitals have all been closed. People's Daily Online, 2020-03-10(02) (in Chinese)

Wen T, Han W, Zhang S (2020). State-owned enterprises in Wuhan show hard core nature to build averagely one mobile cabin hospital every day. Changjiang Daily, 2020-03-20 (in Chinese)

WHO (2020a). Coronavirus disease 2019 (COVID-19) situation report 56. Available at: who.int/emergencies/diseases/novel-coronavirus2019/situation-reports

WHO (2020b). Report of the WHO-China joint mission on coronavirus disease 2019 (COVID-19). Available at: who.int/publications-detail WHO (2020c). WHO Director-General's opening remarks at the media briefing on COVID-19. Available at: who.int/dg/speeches/detail

Zhang H (2020a). Early lessons from the frontline of the 2019-nCoV outbreak. Lancet, 395(10225): 687

Zhang Y F (2020b). "Cabin hospital" model receives praise in virus containment. China Daily Latest, 2020-02-07 